\title{
Fast deutsch, aber nicht ganz... - Fallbeispiel Nr. 4: Ghasem F. (21)
}

Im Frühjahr 2017 lerne ich Ghasem (21) am Eingang des Landesamts kennen. Ich erkläre einer Gruppe afghanischer Geflüchteter, weshalb sie sich in puncto Familiennachzug auch bei Nichtregierungsorganisationen ${ }^{1}$ erkundigen und nicht bloß der Meinung ihrer anwaltlichen Fachkraft Glauben schenken sollten, als sich Ghasem, ein freundlicher junger Mann, dazugesellt. Mittlerweile haben sich anwaltliche Fachkräfte darauf spezialisiert, ahnungslose Asylbewerber*innen hinters Licht zu führen, indem sie ihnen Ammenmärchen erzählen. Die abgelehnten Asylbewerber*innen müssten lediglich jeden Monat fünfzig Euro auf das Konto der Kanzlei zahlen, bis die anwaltliche Fachkraft in unbestimmter Zeit den gesamten Familienverband ihrer neugewonnenen Kundschaft aus dem ländlichen Afghanistan nach Berlin einfliege. Dabei stellt Familiennachzug selbst für anerkannte Asylbewerber*innen eine komplizierte und langwierige Angelegenheit $\mathrm{dar}^{2}$, zumal die meisten Personen derzeit überwiegend aus Syrien nachziehen dürfen. Ich empfehle meinen Gesprächspartner*innen, glaubwürdige Organisationen aufzusuchen und sich von mehr als nur einer anwaltlichen Fachkraft beraten zu lassen. Eine Person ist besonders hartnäckig, sie verlangt Beweise für meine Aussagen. Das könne doch nicht sein, dass im كثنور جهان اول آلمان 3 alle anwaltlichen Fachkräfte in diesem Ausmaß betrügen würden, und dies noch in der Hauptstadt. Ghasem hat ebenfalls Familie außerhalb Deutschlands, was er mir später an demselben Tag erzählt. Wir gehen in den Park gleich gegenüber und spazieren

\footnotetext{
${ }^{1}$ So z. B. Pro Asyl.

${ }^{2}$ Seit August 2018 können pro Monat 1.000 Angehörige in die Bundesrepublik einreisen, wobei für die Auswahl der Personen humanitäre Gründe ausschlaggebend sind. Es besteht demnach kein Rechtsanspruch auf den Nachzug.

${ }^{3}$ „Erste-Welt-Land Deutschland“.
} 
anschließend die Spree entlang. Er musste den ganzen Tag wegen irgendeines Antrages für seine Eltern warten und soll morgen nochmals erscheinen. Er hat Glück, dass er schon ein wenig Deutsch kann, denn für Dari und Farsi ist heute keine übersetzende Fachkraft vor Ort. Ghasem ist sehr kommunikationsfreudig und interessiert, als ich ihm von meiner Studie erzähle. Ich treffe ihn in den folgenden Monaten mehrere Male in Moabit, wo unsere Gespräche stattfinden. Meistens spazieren wir vom Landesamt in ein naheliegendes Café und anschließend wieder an die Spree; oder ich warte mit ihm in den Warteschlangen. Er zieht mich aber meistens förmlich weg von der Behörde, weil er dort keine weitere Minute durchsteht. Zweimal besuche ich ihn in seinem Heim und lerne bei einer der Gelegenheiten die Eltern kennen. Insgesamt treffen wir uns zwölfmal über einen Zeitraum von fünf Wochen, darunter recht viele kürzere Spontanverabredungen.

Ghasem und seine Eltern sind seit knapp anderthalb Jahren in Berlin und leben noch in einer Flüchtlingsunterkunft. Ghasem ist dünn und nicht gerade groß gewachsen. Er hat eine hohe Stirn und relativ kurze Haare. Er spricht schnell und hektisch, schaut während unserer Gespräche wie von selbst etliche Male auf sein Mobiltelefon und klappert mit seinen Fingern Rhythmen auf dem Tisch. Manchmal fällt ihm das auf, dann unterbricht er sein Klappern sofort und schaut entschuldigend drein, während er schmunzelt; manchmal fällt es ihm gar nicht auf und er klopft ein ganzes Konzert. Nach unseren Treffen merke ich, wie seine Nervosität und Verunsicherung mich durchdringen, wenn ich an das Gesagte denke. Schnell wird evident, dass Gefühle der Verunsicherung und Nervosität bei ihm im Vordergrund stehen.

„Ich bin in der Nähe von Kabul groß geworden. Wir sind Qizilbasch. Aus diesem Grund wurden wir immer schon diskriminiert. Wir haben zwar keine Mandelaugen, aber sobald die Leute wussten, dass wir keine Sunniten sind und außerdem Qizilbasch, beleidigten sie uns (...). Afghanistan ist ein Land, das sich schon immer im Krieg befand. Meine Generation hat zum Beispiel jetzt nicht die krassen Kriege von damals miterlebt. Also die Russen und so. Aber trotzdem ist das total bitter in Afghanistan. Zum Beispiel da, wo wir gelebt haben, in Kabul, gab es zwei Schlösser, so wie das Weiße Haus in Amerika, (...) oder das in Berlin, wo die Politiker immer unterwegs sind. Und das eine Schloss ist komplett zerstört worden in Kabul. Die Russen und der Bürgerkrieg haben alles zerstört. Und natürlich dann, als die Amis kamen. Wir durften als Kinder nie dahingehen. [...]

Wir haben halt in einem Viertel gelebt, wo auch ganz viele andere Schiiten waren. Bei Afshar. Das gehört zu Kabul. Die Leute haben uns zu den Tadschiken gezählt, obwohl die Qizilbasch eine eigene Ethnie sind. Die meisten Afghanen sind ja Sunniten. Aber es existieren ganz viele Stämme und Ethnien. Aber die Mehrheit zählt dann einfach alle Schiiten als Tadschiken und macht sich nicht mehr die Mühe, unsere Ethnie einzeln aufzuzählen. Deshalb zählten wir leider zu den Tadschiken. [...]“ 
Ethnische Diskriminierung im Heimatkontext beschreibt Ghasem sehr lebendig, etwa dann, wenn er erzählt, wie er im Kontext der Vorschulprüfungen benachteiligt wurde und erst im Nachhinein begriff, dass nur wohlhabende Menschen, die sich der Taliban beugen, in seiner Heimat eine Chance haben. Mit aufgerissenen Augen und wilder Gestik schildert er mir die Situation an der Grundschule und wiederholt sich in seiner Aufregung ziemlich oft.
„In den Nachrichten haben die das ja wieder gezeigt, wie sie Schiiten angegriffen und getötet haben neulich. Das ist jeden Tag so. Schiiten leben immer in Gefahr. Die merken einfach, dass du Schiit bist. Und dann sind die komisch zu dir. Die meisten Schiiten kommen halt aus Hazarajat und haben Mandelaugen. [...]
Da wir keine Mandelaugen haben, hatten wir uns gut verstecken können im All- tag, aber die haben das trotzdem schnell gemerkt. Und dann haben die sich anders benommen. Außerdem können wir ja kein Paschtu. Das hat man einfach gemerkt. Für jeden, der kein Paschtu kann, ist es schwierig, sehr schwierig da. [...] Aber ist ja egal, ich will jetzt nicht mehr darüber reden."

In den Gesprächen springt Ghasem häufig von einem Thema zum nächsten, ganz nach seiner nervösen Art. Im Café angekommen, bestellt er sich ein Kaltgetränk. Als uns die Bedienung unsere Getränke serviert, zappelt er herum und ist unaufmerksam. Er sucht einen Chatverlauf, den er mir unbedingt zeigen möchte, während er mir nebenbei seine Kindheit zusammenfasst. Einige Augenblicke später nimmt er einen kräftigen Schluck meines kochend heißen Tees in der Annahme, es sei sein Kaltgetränk. Geschockt hält er sich die Hand fest an den Mund und trinkt gleich sein eigenes Getränk hinterher zur Abkühlung. Im Anschluss schüttelt er bloß noch den Kopf, massiert seine Kehle und kommuniziert mit mir, indem er sich fortwährend Vorwürfe macht. Devot entschuldigt er sich mit tausend Worten und Gesten. Er ist derart verwirrt, dass er sich gar nicht mehr beruhigen kann.

„Es ist wirklich so schwer jetzt, die gesamte Lage in Deutschland. Einfach alles. Ich muss meine Eltern betreuen, ich muss jeden Tag Deutsch lernen und meine Ausbildung voranbringen. Das ist sehr schwer. Die ganze Verantwortung zu haben, immer. Ich will ja Menschenrechtsanwalt werden, weißt du? Das war schon immer mein Ding. Menschenrechte. [...] In der Heimat habe ich immer davon geträumt, so frei zu leben, wie die im Westen. Aber es ist echt schwer hier (...).“

Auf Nachfrage, was ihn an den Menschenrechten so fasziniere und welche Rechte er insbesondere meine, kann er außer „Freiheit“ nichts Näheres sagen. Er ist völlig verdutzt, als ich ihm mitteile, dass „Asyl“ ein Menschenrecht ist. Seinen Traumberuf und die damit einhergehende positive Einstellung zur westlichen Demokratie und zum westlichen Lebensstil erwähnt er bei unseren anfänglichen 
Treffen wiederholt. Da er so zahlreiche Termine wahrnehmen muss, schreibt er mir aufs Geratewohl eine Textnachricht, wenn er sich auf dem Weg zum Landesamt befindet. Bei den kurzen, vielleicht fünf bis zehnminütigen Treffen, in denen mit meinen anderen Kontakten Smalltalk angesagt gewesen wäre, berichtet mir Ghasem stolz von seinem Hang zu den Idealen des Westens. Er springt häufig hin und her in den Gesprächen.

\begin{abstract}
„Mein Vater wollte so lange wie möglich in Afghanistan bleiben. (...) Jedenfalls, in den Bezirk, in dem wir gelebt haben, kam eines Tages ein Mörder, Gulbuddin Hekmatyar, ein extremistischer Anführer, der Schiiten bekämpft. Das war vor vielen Jahren. Damals hat er Kabul bombardiert. Bei diesem Angriff sind meine Geschwister gestorben. (...) Seitdem (...) ist alles anders. [...] Ja, und ich will da jetzt nicht darüber reden, das ist wirklich schmerzhaft. Ich will einfach nur die Vergangenheit vergessen. Ich will alles vergessen. Ich frage meine Eltern auch nicht danach. Nur manchmal, wenn sie total traurig sind, sage ich: ,Erzählt mir doch von damals', damit die wenigstens etwas ruhiger werden. [...] Dann erzählen sie von damals. Meistens traurige Begebenheiten, aber wenigsten reden die dann mit mir. Die werden echt noch verrückt. Kein Wunder in diesem Irrenhaus. [...] Stell dir mal vor, mein Vater ist trotzdem noch in Afghanistan geblieben. Nachdem er seine Kinder verloren hat. ,Das ist mein Vaterland, warum soll ich Flüchtling werden? Ich will in meinem Land bleiben', so sprach er immer. Und vor allem, er wusste um die Situation der Afghanen im Iran. Er wusste Dinge, die wir nicht wussten. Als Flüchtling im Iran ist es die Hölle. Aber wir sind trotzdem dahin. Das war der Fehler. [...] Seitdem hat unsere Misere angefangen. Was für eine badbakhti ${ }^{4}$. Aber ich werde meinem Vater nicht so schnell vergeben, dass wir nicht früher abgehauen sind. (...) Meine Geschwister wären jetzt sicher am Leben.“
\end{abstract}

Manchmal macht er mitten im Gespräch zuckende Bewegungen mit der Schulter und guckt sich Gegenstände an, die weiter weg stehen und gar nichts mit dem gerade Besprochenen zu tun haben. Dabei pickt er sich dauernd die Haut von den Lippen und mustert diese Dinge, etwa eine Vase im Café, die auf der letzten Fensterbank steht, äußerst genau. In diesen Momenten scheint er für ein paar Sekunden mental verschwunden zu sein. Wenn er zu sich kommt, wartet er gar nicht ab, was ich wissen möchte. Er erzählt einfach.

„Am Anfang mussten wir Fingerabdrücke abgeben und wurden ein paar Mal abfotografiert. Wie die Tiere, echt. (...) Im Camp haben die uns ohnehin behandelt wie Verbrecher. Das habe ich auch nicht verstanden anfangs. Ich dachte, hier ist der Ort der Menschenrechte, ich dachte, hier sei der Himmel. [...] Mein Vater war von

4 بدبختى - Misere, Elend, Armseligkeit, Not; vgl. Fallbeispiel Nr. 1. 
Anfang an misstrauisch, wenn ich von meinen Wünschen erzählte, auch wie die Westler zu leben. Jetzt verstehe ich auch, warum. Wir mussten die ganze Zeit hinund herziehen. Und im letzten Camp, ach. [...] Dann durften wir nicht mal Besuch bekommen, meine Eltern hatten nämlich unterwegs ein Ehepaar kennengelernt, die sie gerne öfter gesehen hätten. Sie kamen dann auch irgendwann zu Besuch. Aber du glaubst ja nicht, was die für einen Aufstand machten. Alter Schwede! ${ }^{5}$ Und dann noch das mit der Hähnchenkeule an diesem verfluchten Festtag. [...] Großer Gott! [...] Komm, lass uns das Thema wechseln.“

Ein anderes Mal besuche ich ihn im Camp. Nachdem die streng dreinblickenden Sicherheitskräfte an der Rezeption des Hotels meinen Ausweis kontrolliert und eingesammelt haben, darf ich mit Ghasem rein. Das Hotel wurde wie etliche andere Hotels in Berlin zu einer Notunterkunft umfunktioniert. Ghasem ist übrigens ebenfalls verpflichtet, seinen Heimausweis vorzuzeigen, mit dem er bereits seit unserer Haltestelle herumspielt. Als wir mit dem Aufzug hochfahren, quetschen sich noch ein halbes Dutzend Personen in den Aufzug, vollbepackt mit Lebensmitteln vom Wochenmarkt. Ghasem und ich werden höchst kritisch von ihnen gemustert. Dann beginnen sie auf Arabisch zu spotten und verachtend auf uns zu schauen. Der Familienvater, der die Verspottung vor seinen Kindern anführt, isst unterdessen geröstete Sonnenblumenkerne und spuckt deren Schale nach jedem Mal vor sich hin. Die Mutter bestätigt mit ihrem Lachen seinen Spott, woraufhin die Kinder einsteigen. Als wir schließlich die Aufzugsituation verlassen dürfen, muss noch eine Glastür überwunden werden, bis wir im richtigen Gang sind. Der Geruch von Urin schwebt im Korridor, den wir entlangschreiten; am Ende des Flurs scheinen die Gemeinschaftssanitäranlagen zu liegen. Geschrei und Gezänk unterschiedlicher Parteien sowie Geräusche verschiedener Medien - Handys, Radios, Liveübertragungen von Videotelefonie und arabische Musik - beschallen uns. Ich bin auf jeder sinnlichen Ebene, visuell, olfaktorisch, haptisch und akustisch, dem Getöse und Schmutz des Heims ausgesetzt. Im Zimmer sieht man die liebenswerten Versuche, mit kleinen Decken und Teppichen aus der Heimat ein Gefühl der Vertrautheit entstehen zu lassen. Ghasems Eltern sind nicht da. Sicherlich sind sie aus Höflichkeit, im Geiste des ta'arof, weg, um das kleine Zimmer, das sich die Familie teilt, nicht unnötig zu füllen. Obwohl der Raum ordentlich und frisch geputzt ist, kriecht hin und wieder der Gestank von draußen herein. Doch noch schlimmer als dies empfinde ich die Tatsache, dass wir jedes Wort und jeden Atemzug des heftigen Streits

اي بابا5 
von nebenan miterleben. In der Ecke steht ein umständlicher und kitschiger Samowar, den Ghasems Vater, wie ich erfahre, von einem Berliner Flohmarkt erstanden hat. Ghasem springt servierfreudig im Zimmer herum und bringt mir einen Snack nach dem andern. Er ist gleichermaßen herzlich wie aufgekratzt. Im Zimmer sind unzählige Tüten und Taschen mit Blättern, Akten und Dokumenten darin. Auf Ghasems schmalem Bett befinden sich Formulare, die er für seine Eltern ausfüllen muss, Kopfhörer, Bollywood-DVDs auf Dari, ein Beutel voller rosa-weißer afghanischer Süßigkeiten - marshmallowartige Quadrate mit Pistazien, die überhaupt nicht schmecken, die ich jedoch aus ta'arof-Gefühlen nicht ablehnen kann - und das A2-Kursbuch mit dem dazugehörigen Übungsheft. Auf dem kleinen Schränkchen am Bett stehen lauter Tabletten und kleine Dosen, die Pillen enthalten. Auf dem Tisch sieht man prachtvoll geschmücktes Obst auf einer Silberschüssel. Mittlerweile artet der Streit der Nachbarn aus und man hört Security-Personal anmarschieren. Neben dem Arrangement, bestehend aus Granatäpfeln, Bananen, Datteln, Äpfeln und Aprikosen, steht eine Schüssel randvoll mit Pistazien und anderen Nüssen. Auf dem einzigen Regal steht der Koran. Man sieht orientalische Muster und Farben überall zwischen den eintönigen und tristen Wänden und Formen verteilt, so dass das trostlose Zimmer beinah charmant wirkt. Die kreativen Versuche, sich im Ankunftskontext affektiv zu verorten, lese ich als eine Spielart der ,place-making practice“ mit dem Ziel der Heimatbildung (Röttger-Rössler 2016: 19). Sogenannte „place-making practices" beziehen sich auf Strategien und Praktiken, mit deren Hilfe Asylbewerber*innen bzw. Migrant*innen versuchen, sich affektiv an neue Orte oder simultan an mehrere gleichzeitig zu binden (ebd.). Die Versuche, in Extremsituationen, wie hier in Massenunterkünften, Heimatbildungsprozesse durch das Erleben vertrauter Eindrücke - charakteristischer Farben, Muster, Gerüche, Klänge und Formen - auf der sensorischen Ebene herzustellen, scheinen darauf zu zielen, die fremde Umgebung den verkörperten Wahrnehmungsmodi, dem sogenannten „haptic habitus“ (Wise 2010), anzupassen. Damit kann ein Stück Vertrautheit generiert werden. Während uns die von außen kommenden Geräusche mit ihrer Penetranz zu nerven beginnen, spüre ich, wie Ghasem sich für diese schämt. Kaum schlage ich vor, in den naheliegenden Park zu gehen, um dort zu flanieren, steht Ghasem bereits an der Tür und schnürt sich die Schuhe zu. Im anschließenden Gespräch beim Spaziergang geht es um die eben verbrachte Zeit im Heim. Auf konkrete Nachfrage bestätigt er meinen Verdacht. Er schämt sich, wenig verwunderlich, für seinen Lebensraum. Außerdem ist ihm unangenehm, dass wir im Aufzug von fremden Menschen verhöhnt worden sind. Hinzu kommt die Lautstärke der Nachbarn im Zimmer, die ausgerechnet heute Streit haben mussten. Er schaut mir niemals lange in die Augen und spricht in 
einem unangenehm schnellen Tempo. Wenn ich ihn etwas frage, spricht er manchmal ohne Punkt und Komma, an anderen Tagen deutlich weniger und noch unzusammenhängender als sonst. Bisweilen ist sein Dari, angesichts der Geschwindigkeit und seines speziellen Dialekts, für mich nicht mehr verständlich. Teils mischt er sogar neu erlernte englische oder deutsche Begriffe oder vielmehr Halbsätze in das Gesagte, was die Sache nicht einfacher macht. Nach unseren intensiven Treffen bin ich erschöpft, nervös und fühle mich in Bezug auf Dinge, die mich normalerweise nicht ins Schwanken bringen, verunsichert. Auftretende Affekte, vor allem die nervösen, begreife ich als epistemologische Hinweise darauf, dass die Gefühle in Ghasems Lebenswelt in meinem Gemüt reflektiert werden. Die nervöse, hektische, aufgescheuchte Art und Weise beim Reden und Handeln von Ghasem erzeugt eine Spannung und beeinflusst unsere Begegnungen stark. Bei unserem letzten Treffen verkündet er, nachdem ich ihm noch einige Verständnisfragen hinsichtlich seiner Familiengeschichte stelle, Folgendes:

„Wenn ich endlich Geld verdiene und einen Job habe, schickt mir die Verwandtschaft aus der Heimat eine Frau nach Deutschland.“

Auf Nachfrage kann er jedoch keine konkreteren Angaben machen, da diese Angelegenheit von seinen Verwandten geplant wird. Er weiß lediglich, dass die Braut in Kürze nach Istanbul gefahren werden soll. Dort soll Ghasem sie heiraten und mit ihr nach Berlin fliegen. Er ist bei diesem Treffen kurz angebunden und möchte unser Gespräch nach fünfzehn Minuten beenden. Nach dem letzten kurzen Treffen verlieren wir den Kontakt, weil ich ihn nicht mehr erreichen kann.

Ghasem fühlt sich ethnisch den Qizilbasch zugehörig. Seine Ethnizität ist eines unserer Einstiegsthemen der Gespräche. Die Qizilbasch gelten als Nachfahren der turkmenischen Safawiden, die im späten 11. Jahrhundert nach Afghanistan einwanderten (Mohebbi 2011: 17). Sie sind ethnisch keine homogene Gruppe (Puzhohish 2005: 9). Der Begriff Qizilbasch wird teils für die vielen schiitischen Religionsgruppen verwendet, die sich seit dem späten 13. Jahrhundert in Anatolien und Kurdistan ausbreiteten. Ghasem beschreibt ausführlich, wie sich die Benachteiligung konkret in ihrem Leben ausgewirkt hat und wie sehr sie sich glücklich schätzen könnten, dass sie keine „Mandelaugen“ hätten. Die Diskriminierung von Schiiten seitens der sunnitischen Mehrheit ist prägend für seine Persönlichkeit. Ghasem lernt sehr früh, dass die eigene Ethnizität und Religion im öffentlichen Leben verheimlicht werden muss, um keine soziale Ausgrenzung zu erfahren. Doch es bleibt nicht nur bei Ausgrenzungen im kriegsverzehrten Afghanistan, denn sie werden in die Flucht getrieben. Nach den traumatischen Erlebnissen des Todes seiner Geschwister durch einen Bombenangriff befindet 
sich die Familie im Ausnahmezustand. Sein Vater kennt die Leiden eines geflüchteten Menschen und vermeidet bis zur letzten Sekunde die Flucht aus Afghanistan. Ghasems Groll auf seinen Vater rührt daher, dass er ihm die Schuld für den Tod seiner Geschwister gibt. Als es beinahe zu spät ist, beauftragen sie Schlepper, die sie mit ihrem Haus bezahlen, und flüchten in den Iran. Dort bleiben sie nicht lange und erreichen binnen zehn Tagen Europa. Seitdem redet seine Mutter kaum noch mit ihm. Alles, was sie zum Besten gebe, seien Floskeln und Wendungen wie ,Junge, das schaffst du schon!“. Ghasem fühlt sich entfremdet. Seine Belastung wurzelt in der enormen Verantwortung, die ihm im Ankunftskontext übertragen wurde und die er nicht ohne Weiteres stemmen kann. Seine Eltern erwarten, dass er sämtliche amtlichen und behördlichen Angelegenheiten der Familie selbstständig klärt. Sie selbst scheinen handlungsunfähig und wälzen die gesamte Verantwortung auf den Sohn ab. Beispielsweise erscheinen sie nicht zu ihren Terminen, weshalb Ghasem alle paar Tage an irgendeiner Stelle der Institution Asyl vorstellig werden muss, jedes Mal mit einem Berg von Attesten, Bescheinigungen und Akten. Die traumatischen Erlebnisse der Angriffe, bei denen die beiden Geschwister getötet wurden, werden selten thematisiert. Kommt es doch vor, fühlt er sich in der Rolle eines ,Therapeuten“, indem er sich bemüht, die Eltern „von damals“ erzählen zu lassen. Dies passiert gelegentlich, wenn es ihnen äußerst schlecht geht und sie beleidigt in ihrem Zimmer sitzen. Ghasems Vater versteht sich als Patriot und stolzer Qizilbasch, der nun am Tiefpunkt seines Lebens angelangt ist und resigniert. Allein die Tatsache, dass sie vom deutschen Staat erhalten, ist ihm von Anfang an ein Dorn im Auge. Gemeint ist die monatliche Sozialhilfe, die gemeinhin als حقوق übersetzt wird, weil sich das Konzept der Sozialhilfe, wie sie in Deutschland verstanden wird, nicht oder sehr schwer in den Farsi- bzw. Dari-sprachigen Kontext übertragen lässt. In Ghasems Heimat überleben sozial schwache Personen mehrheitlich durch „Almosen und Spenden“ und nicht mittels „,sozialer Absicherung“. Dies sorgt für Verwirrungen, nicht nur bei Ghasems Familie. Viele Asylbewerber*innen fragen sich, weshalb sie ein Monatsgehalt erhalten, ohne arbeiten zu müssen, und empfinden dies als ausgesprochen ideal, während andere sich beleidigt fühlen und vereinzelt sogar Geld- oder Sachleistungen ablehnen. Das fehlende Gefühl der Zugehörigkeit ist ein oft wiederkehrendes Gesprächsthema.

\footnotetext{
${ }^{6}$ Der Begriff hat zwei Bedeutungsdimensionen, zum einen Lohn, Gehalt, Entgelt, Entlohnung, Verdienst, zum anderen ist حقوق der Plural von Recht. In diesem Zusammenhang ist die erste Bedeutungsebene gemeint, also etwa das monatliche Gehalt eines Familienvorstandes.
} 
Als Minderheit wurde Ghasem immer schon diskriminiert und empfindet es als „Glück“, keine „Mandelaugen“ zu haben. Er besucht einen Sprachkurs und einen Ausbildungsvorbereitungskurs. Die Richtung der Ausbildung ist noch nicht eindeutig. Ghasem gibt zu verstehen, dass er zunächst die nächstbeste Ausbildung beginnt, um später noch zusätzlich einen Abschluss zu absolvieren. Dies ist charakteristisch für ihn. Er plant sehr viel und redet mit großer Sicherheit über Dinge wie das Jurastudium, die zunächst wenig realistisch scheinen und betont seine Zuneigung zu westlichen Werten und Menschenrechten. Gleichzeitig drückt er seine Verunsicherung unmissverständlich aus - die hektischen Bewegungen des Knies, das plötzliche Kratzen an diversen Körperstellen und das Zerreißen seiner Lippen sind Teil der unbewussten Inszenierung seiner sinnlichen Metamorphose. Ghasem berichtet davon, wie seine Eltern Bekannte zum Abendessen einluden, obwohl keine Erlaubnis vorlag, zumal die Zeit laut Unterkunft für einen Besuch unpassend war. Es gab anschließend einen heftigen Streit vor der Unterkunft, insbesondere weil Ghasems Vater sich in seiner Ehre gekränkt fühlte. Am Qizilbasch-Feiertag, ,schmuggelte“ seine Mutter Fleisch in die Unterkunft, um etwas Festliches auf dem Tisch zu haben. Das Kochen auf den Zimmern ist jedoch untersagt. Als seine Mutter mit dem Hähnchen unter ihren Achseln „erwischt“ wird, ist die Blamage vor den Freunden und anderen Insassen perfekt. An dieser Stelle wird deutlich, wie perfide solche Dynamiken wirken und welche Bedeutung die Bevormundung für Emotionen im Asylprozess hat. Die übermäßige Kontrolle des Besuchs und die ausartenden feindseligen Reaktionen zwischen einzelnen Akteur*innen werden als Spielarten einer Beschränkung der „Territorien des Selbst“ (Goffman 1974) innerhalb der Institution gedeutet. Diese Restriktionen betreffen die soziale Ordnung des Raums. Der Raum, oder besser die totale Kontrolle des Körpers von Ghasem im Raum, entmündigt ihn und trägt zur affektiven Konstitution seines Selbst bei. Vormundschaft und Kontrolle über Räume, gleichsam soziale Räume, wie das Beispiel Ghasems veranschaulicht, gehen in totalen Institutionen Hand in Hand. Essenzielle Fragen, Aktivitäten und Themen des Lebens wie Wohnen, Freizeitgestaltung, Ernährung, Sexualität und Partnerschaft stehen unter dem Dirigat der Institution. Das totalitäre Merkmal der Entmündigung stellt den grundlegenden Akt dar, der in den Zustand der Bevormundung resultiert (Gallati Zimmermann 2012: 198) und die sozialen Interaktionen (Katz II) Ghasems entscheidend beeinflusst. Welcher Art diese Kränkungen auch sein mögen, das gefangene Individuum ist genötigt, ein Verhalten zu zeigen, dessen symbolische Implikationen mit seiner Vorstellung von sich selbst unvereinbar sind (Goffman 1973: 32). So gestaltet sich in einem spannungsvollen Raum die Identitätszuweisung des Gefangenen und trägt Züge einer Selbstunterwerfung. Der Versuch, an einem Festtag etwas von Bedeutung zu 
zelebrieren und zu performieren - bei der genannten Festlichkeit geht es um einen symbolischen „Neu-Anfang im Leben“, mehr erzählt Ghasem nicht darüber -, ist zugleich ein Versuch, durch ein soziales Ritual Gefühle ,inter- und intrapersonell zu kommunizieren“ (Röttger-Rössler 2004: 176). Insbesondere angesichts des Stresses und der Spannungen im Dritten Raum sind soziale Rituale von Relevanz. In diesem Moment wird erneut die Totalität der Machtstruktur in der Institution deutlich, die durch Kontrolle des sozialen Raums in soziale Interaktionsprozesse des Selbst eingreift. Hinzu kommt der Wunsch, die Zugehörigkeitsgefühle unter den einzigen Vertrauten der Familie zu stärken, die derselben Ethnie angehören. Die Wichtigkeit dieses Ereignisses und die entsprechende Intensität der Blamage vor den Freunden, Nachbarn und anderen Heimbewohner*innen lassen Ressentiments aufsteigen. Seitdem pflegen die Eltern Ghasems keinen Kontakt mehr mit ihren Freunden und isolieren sich gänzlich. Nach den erschütternden Erlebnissen und unter dem aus ihnen entsprungenen Druck willigt Ghasem ein, das schiitische Mädchen aus Afghanistan zu heiraten, sobald er über eine feste Arbeitsstelle verfügt und der Aufenthalt geregelt ist. Die Angst vor einer Abschiebung spielt demnach keine große Rolle mehr, insbesondere nachdem mehrere Seiten (etwa Sozialarbeiter*innen im Heim) bestätigt haben, dass abgelehnte Asylbewerber*innen nicht zwangsläufig abgeschoben werden müssen. Mit der Inszenierung seines sozialen Dramas als hypernervöser Musterjunge, der sein gesamtes Leben den Eltern widmet, passt Ghasem sich den Bedingungen der totalen Institution Asyl an. Dabei sind verschiedene, teils unvereinbare Facetten seiner Inszenierung hervorzuheben. Einerseits spielt er die Rolle des guten, braven Sohnes, der auf seine Eltern hört und das ausgesuchte schiitische Mädchen aus Afghanistan heiratet. Damit drückt er nach meiner Interpretation seine Zuneigung für seine Eltern aus. Durch den Verheiratungsplan werden zudem die Bande zwischen ihm und seinen Eltern wiederbelebt. Er bietet sogar Gelegenheit, neue Allianzen mit Verwandten zu schmieden. All diese Elemente des sozialen Dramas zielen darauf $a b$, der verunsichernden Dynamik des Identitätsverlustes sowie der Inferiorisierung im Ankunftskontext entgegenzuwirken.

Die beschriebenen typischen Emotionsausdrücke (sein „nervöses Drama“) werden als Signale des „existenziellen Gefühls“ (siehe Abschn. 4.2.4) der Verunsicherung gedeutet. Aus Sicht der Emotionstheorie Ratcliffes liegt die alltägliche Erfahrung des Emotionen erlebenden Menschen in einem Meer von miterfassten Möglichkeiten der Handlungsmächtigkeit und des zwischenmenschlichen Umgangs. Alle Möglichkeiten des Interagierens mit anderen Personen und Gruppen sind beim inferiorisierten Menschen beeinträchtigt. Aus dieser Beeinträchtigung heraus resultiert ein Gefühl der Spaltung und Befremdlichkeit. Ghasem findet keinen authentischen Bezug zur deutschen Gesellschaft, obwohl er sich 
dies zu Beginn wünschte. Die fehlende Zugehörigkeit in der neuen Gesellschaft spielt mit Blick auf die von der Familie vorgeprägte Entscheidung, ein afghanisches Mädchen einfliegen zu lassen und $\mathrm{zu}$ heiraten, eine maßgebliche Rolle für seine Lebenswelt. Die Gesamtstruktur seines personalen und interpersonalen Seins kondensiert sich im existenziellen Gefühl, das durch Verunsicherung, Ungewissheit, Zweifel und vor allem Zwiespalt konstituiert wird. Dieses wird zum strukturbildenden Medium seiner Erfahrungen (Slaby 2012: 84). All die Elemente, die zum Emotionsausdruck gehören (Baustein III des Emotionssystems), legen die Verunsicherung offen und machen sie unmissverständlich deutlich. Ghasem kann seine Nervosität und Unsicherheit nicht abstellen, weil sie den Rahmen jeglichen Erlebens in seiner Lebenswelt bilden. Erhoffte Gefühle der Sicherheit und des Vertrauens bleiben im Ankunftskontext aus. Der Wunsch, ,auch, wie die Westler zu leben“, bekommt einen Riss. Die in diesem Beispiel beschriebenen existenziellen Gefühle verdeutlichen die Transpersonalität des Ratcliff'schen Ansatzes. Die Subjekt-ObjektTrennung löst sich auf, aber nicht gänzlich, so dass die existenziellen Gefühle weder vollständig auf den in der Institution gefangenen Menschen noch auf die totale Institution zurückzuführen ist; sie sind gewissermaßen dialogisch strukturiert. Passend zu den sozialphänomenologischen (Abschn. 2.2) und subjekttheoretischen (Abschn. 4.6) Positionen dieser Studie können die affektiven Weltbezüge Ghasems nicht getrennt vom existenziellen Gefühl der Verunsicherung und umgekehrt Ghasem nicht ohne seine affektiven Weltbezüge begriffen werden (vgl. Ratcliffe 2008: 234 f.). Die Spaltung von Subjekt (Selbst) und Objekt (Welt) verbindet sich auf einzigartige Weise im Konzept der existenziellen Gefühle, da die Welt bereits affektiv erschlossen und mit der speziellen Färbung des jeweiligen existenziellen Gefühls versehen wurde. Der gefangene Mensch kann demgemäß nicht sinnvoll gedacht werden ohne seine spezifischen Affekt-Weltbezüge. Die Stärke von Ratcliffes Ansatz zeigt sich hier im Gesichtspunkt der Erfahrungsdimension Ghasems. Diese Dimension kann in seiner Lebenswelt beleuchtet werden, ohne unnötigen Psychologisierungen und Überzeichnungen von Charakteren anheimzufallen. Demnach wird eine transzendentalphilosophische Sicht etabliert, die den basalen menschlichen Weltbezug im Anschluss an Heidegger beschreibt (Slaby 2012: 80). Die fehlende Systematik der Theorie existenzieller Gefühle lässt sich mit einer Einteilung in belastbare Kategorien von Hintergrundgefühlen kompensieren, die allerdings nur in einer empirischen Forschung ans Licht treten können, weil das Subjekt nicht auf seine eigenen Hintergrundgefühle zugreifen kann. Die methodologische Herausforderung besteht daher darin, zuverlässige, intersubjektiv nachvollziehbare Narrative über Hintergrundgefühle zu erlangen, um veränderte Erfahrungsformen besser nachvollziehen zu können. 
Die im Theorieteil erwähnten Aufnahmerituale (vgl. Abschn. 4.1), etwa das Aufnehmen der Fingerabdrücke und das Fotografieren während der Registrierung oder das Zuweisen von Schlafplätzen beim Eintritt in totale Institutionen, sind als Teil jener Prozeduren zu verstehen, die den bürgerlichen Tod Ghasems bezwecken. Das Erfassen und Sortieren messbarer Daten in den genannten Formen sowie vor allem die nunmehr modifizierte Zuschreibung seiner Identität ist vor dem Hintergrund der impliziten Annahme über das Wesen des Menschen innerhalb der Institution zu sehen. Ghasem rutscht mit dem Eintritt in die Gemeinschaft der registrierten Asylbewerber*innen in die abstrakteste Kategorie des Sozialen, ,der Menschheit“ (Neumann et al. 2003: 382). Damit rückt Ghasem ein Stück mehr von seiner ehemaligen Identität ab. Das Verhältnis zwischen dem Gefangenen und seinen Handlungen wird auf diese Art systematisch erschüttert. Seine Handlungsmöglichkeiten werden durch den Erziehungsgeist der Institution Asyl in Form von Sanktionen und Belohnungen gesteuert (vgl. insbes. Fallbeispiel 6), teils unbeabsichtigt, wie ,automatisiert“ (vgl. Fallbeispiel 5). Aus dieser Situation herauszutreten ist schlicht nicht möglich, da Ghasem totalitären Dynamiken ausgesetzt ist. Die haftähnliche Situation in seinen Unterkünften verdeutlicht den gegenwärtigen Diskurs zu Fluchtmigrierenden und etabliert deren Repräsentation. Geflüchtete Menschen müssen demnach grundsätzlich in einer "Sicherheits- und Schutzzone“ leben. Ghasems existenzielle Gefühle - Verunsicherung, Ungewissheit, Zweifel und Zwiespalt - sind auch als Folge dieser Repräsentationspolitik zu deuten. Letztere trägt entscheidend zum medial produzierten Fremd- und Feindbild vertriebener Menschen bei.

Die hierdurch erlebte Entwürdigung beeinflusst Ghasems Identität (Subjektwerdung). Mit Butler lässt sie sich als diskursiver und begrifflicher Besitz der Institution Asyl verstehen. Zentral im Identitätsfindungsprozess ist das Moment der diskursiven Anrufung, der sogenannten Interpellation (vgl. Abschn. 4.6): Indem Ghasem ihren Gehalt annimmt und verinnerlicht, bestätigt er die diskursive Konstruktion über sich und damit seine eigene Ausschließung. Seine Eigen- und Fremdwahrnehmung wird über die Annahme der Anrufung strukturiert. Er wird derart angerufen, dass er sich nach vorliegender Interpretation nicht ernst genommen fühlt und seinen Enthusiasmus für die eigene Integration in die deutsche Gesellschaft verliert. Ghasems Identität ist vielfachen Verunsicherungen ausgesetzt, bis er seine Begeisterung für den neuen „westlichen“ Lebensstil in Deutschland verliert. Von großer Wichtigkeit ist hierbei die als unglaubwürdig bewertete Fluchtgeschichte der Familie, die letztlich zu seinem schwachen Schutzstatus führt. Die Entwurzelung von der Heimat, die Trennung von Freunden und Verwandten und die permanente Konfrontation mit der spezifischen Art der Interpellation sind ebenfalls zu nennen. Ghasem erkennt im Verlauf des Asylprozesses auf schmerzhafte Weise 
die Gespaltenheit des Menschenrechts auf Asyl, das er nach vorliegender Deutung als scheinheilig begreift. Er ist jetzt ebenfalls gespalten. Er bindet sich nun an Vorstellungen, Werte und Traditionen, die ihm Sicherheit und Halt geben. An dieser Stelle offenbart sich ein Zwischenraum, in dem er spielerisch und uneindeutig mit Differenzen spielt.

Ghasems soziales Drama, das die beschriebene Ambivalenz widerspiegelt, wird als unbewusste Erwiderung auf die gescheiterte soziale Anerkennung interpretiert. Seine sinnliche Metamorphose drückt sich in den beschriebenen Emotionsausbrüchen aus und verfestigt sich allmählich aufgrund des sich wiederholenden verkennenden Musters seiner sozialen Interaktionen. Ghasems körperliches Gefühl wandelt sich in der Konsequenz (Katz I) und wird zum Träger seiner stressbehafteten Erfahrungen, die sich aus der Resonanz der Interaktionen (Katz II) speisen. Sein aufgewühltes Verhalten und seine Entscheidung, ein Mädchen aus der Heimat zu heiraten, sind in der vorliegenden Deutung nicht bloß „,negativ“ konnotierte Handlungsweisen, sondern eine kreative und performative Leistung zur Konstitution einer spezifischen Narration (Katz III), die seiner bedrückenden Situation als Gefangener in der Institution Asyl neuen Sinn verleiht. Die mit seiner Narration einhergehenden existenziellen Gefühle spielen eine zentrale Rolle im Unterwerfungs- und Identitätsbildungsprozess. Die Kontrolle über die Platzierung seines Körpers im Raum sowie die aus der Beschränkung seiner Handlungen - Kochverbot, Besuchsverbot, Residenzpflicht erwachsende Entmündigung tragen ebenfalls zu dem Gefühl, „ein Verbrecher“ $\mathrm{zu}$ sein, bei. Ghasem ist diesen Reglementierungen ausgesetzt. Eine weitere Seite der Ausgesetztheit spiegelt sich in dem Umstand wider, dass Bedienstete unentwegt die Insassen kritisieren (Neumann et al. 2003: 382), die ihrerseits permanent im Modus der Rechtfertigung denken, sprechen und handeln (Fragehoheit-Rechtfertigungsangst; siehe Fallbeispiel Nr. 2). Damit wird über die Zerstörung der Autonomie des Handelns auch gezielt auf das Denken und Fühlen Ghasems eingewirkt. Ein bedeutsamer Aspekt der sozialen Interaktionen zeigt sich darin, in Unterkünften für Geflüchtete Spott und Hohn ausgesetzt zu sein. Die auf diese Weise erfolgte Ausgrenzung desintegriert Menschen, spaltet sie und wirkt systemstabilisierend auf die Institution Asyl. Durch jeden Gesichtspunkt seines Lebens innerhalb der Institution wird „organisierte Desintegration“ (Täubig 2009) hervorgerufen. Folglich lebt Ghasem nicht das westliche Leben mit all seinen freiheitlichen Werten aus, wie ursprünglich beabsichtigt, und verhindert mit seiner Narration die eigene Integration in die deutsche Gesellschaft. Der geschilderte Ehrverlust am Eingang der Unterkunft kann als affektive Initialzündung für das Verblassen seiner Affinität zum westlichen Leben und seines Wunsches, Menschenrechtsanwalt zu werden, interpretiert werden. Denn 
in dieser Situation war er einem übermächtigen Feind hilflos ausgeliefert. Er war in der Situation mehr oder minder chancenlos. Die durch die Peinlichkeit potenzierte Kränkung endete in einem Zustand beschämender Ausgesetztheit, was einer emotionalen Traumatisierung gleichkommt.

Auf der untersten Stufe des Selbst angekommen, bilden Scham oder vielmehr „Schande“ (Berrenberg 2002: 82; Möwe 1998: 496) und Wut das Movens des Vorhabens, sich vom ursprünglichen Ideal der westlichen Gesinnung abzuwenden. Spätestens nach diesem Ereignis realisiert Ghasem, dass Deutschland doch ,nicht der Himmel“ ist, von dem er vor seiner Ankunft träumte. Die Totalität der Institution Asyl beeinträchtigt seine Emanzipation und bewirkt die partielle Abkehr von der deutschen Gesellschaft. Die Hybridität, die im Zuge der Narration Ghasems aufkeimt, lässt Anpassungsstrategien aufblühen, die die Fiktionalität vorgegebener Identitätsmuster des Ankunftskontextes zum Vorschein bringen. Bei Ghasem zeigt sich dies in Form einer "geheimen Kunst der Rache“ (Bhabha 1997: 112), quasi als unwillentlich ausgeführte Entgegnung auf die ungerecht empfundene Unterdrückung und deren Strategien und Konsequenzen. Er akzeptiert die unterlegene Rolle (,Knecht“), erfüllt jedoch die „Vorgaben“ dieser Identität nicht ganz. Er möchte sich integrieren und ,wie ein Westler leben" (fast deutsch ...), zugleich schützt er sich vor weiteren Angriffen, indem er eine ganz und gar ,unwestliche“ Beziehung eingeht (... aber nicht ganz). Die Hybridität seiner Subjektwerdung offenbart zudem die kulturelle Vielstimmigkeit des Dritten Raums, die jegliche Formen totalisierender Vereinnahmungen unterbindet - eine Vielstimmigkeit, die Inkommensurabilitäten, Widersprüche und kulturelle Differenzen zulässt, ohne sie in einer absoluten Gesamtheit aufzulösen.

Um langfristig die Andersheit „der jeweils Anderen“ nicht zu verleugnen, ist es notwendig, über das dialektische Denken hinauszugelangen und die „tiefgreifenden Beschränkungen eines auf Konsens und Komplizenschaft beruhenden ,liberalen ' Begriffs von kultureller Gemeinschaft neu zu überdenken“ (Bhabha 2000: 261). Die vorliegende Sichtweise demonstriert unmissverständlich, dass Identitäten, sowohl kulturelle als auch politische, immer im Prozess der Alterität und auf der Basis von Differenz konstruiert werden (ebd.). Die sogenannte Assimilation von Minderheiten an holistische Anschauungen und Denkweisen kultureller Werte ist für das Nachvollziehen von Ghasems ambivalenten Gefühlen gegenstandslos. Mitten in der deutschen Hauptstadt können geflüchtete Menschen Teil der westlichen Gesellschaft werden und zur selben Zeit Ansichten verfolgen, die dem demokratisch-westlichen Wertehorizont diametral entgegenstehen. In seiner Analyse von Nation argumentiert Bhabha im Hinblick auf solche Differenzen: „The ,other" is never outside or beyond us; it emerges forcefully, within cultural discourse [...]“ (Bhabha 1990: 4). Die „Andersheit“ Ghasems 
wird also nicht einfach in ,kultureller Pluralität“ aufgelöst. Unter diesem Gesichtspunkt können die fiktionale Konstruktion des „Deutschseins“ und damit assoziierte Vorstellungen kultureller Homogenität dekonstruiert werden. Widersprüchliche Kulturen stehen demnach nicht gleichberechtigt nebeneinander (Multikulturalismus); sie sind aufgrund der Diskursivität von Kulturen in ihrem eigenen Inneren verortet (ebd.).

Ghasems hybride emotionale Erfahrung ist gleichsam das Sinnbild der spannungsvollen Ambivalenz zwischen unterschiedlichen kulturellen Vorstellungen. Solche Formen der Hybridität dekonstruieren die ,große Erzählung“7 der Einheitlichkeit, ,Reinheit“ und Autorität von Kulturen (Bhabha 1990: 218) und erschüttern die Selbstdefinition der dominanten Gesellschaft - besonders wenn im deutschen Kontext die Konstellation „muslimisch“ vs. „nicht muslimisch“ hinzukommt. Doch hier geht es genau genommen nicht um religiöse Inhalte, sondern um Emotionen, mithin um Ressentiments. Sie werden herangebildet und lassen im Dritten Raum einen Nährboden für unlösbare Widersprüche entstehen, der Desintegrationsprozesse begünstigt. Die bevorstehende arrangierte Ehe Ghasems, zumal mit einer minderjährigen Person (zum Zeitpunkt des Gesprächs soll sie sechzehn Jahre alt gewesen sein), zieht die westliche Gesellschaft insofern in Zweifel, als sie den freiheitlichen Kern des westlichen Lebenskonzepts verfremdet. Das Individuum, das sich frei von Klasse, Gender, Ethnie und Religion Lebens- bzw. Ehepartner*innen suchen und ihre*seine spezielle Art zu leben ohne Verfolgung und Diskriminierung ausleben kann also genau die Art von Lebensstil, die sich Ghasem zu Beginn seines Aufenthalts wünschte -, wird durch seine eigene Narration infrage gestellt. Von diesem Standpunkt aus gesehen bedeutet Hybridität nicht so sehr, ,teils afghanisch, teils deutsch" zu sein, sondern es geht vielmehr darum, dass diese Teile antagonistisch und agonistisch miteinander und zugleich mit dem dominanten Diskurs interagieren (Bhabha 2012: 66). Kulturelle Hybridität stellt sich aus dieser Perspektive als ein Prozess dar, dessen Antrieb sich wiederholende Affekte und existenzielle Gefühle bilden, die den empfindenden Menschen ein spezifisches Drama kreieren und performieren lassen. Die Verhaltensweisen, die im Rahmen dieser Narration an den Tag gelegt werden, treten als Momente der Transformation emotionaler Prozesse auf, durch die die Beschaffenheit und das Wesen sozialer Interaktionen des Ankunftskontextes registriert, gedeutet und zugleich ko-konstruiert wird. Die Hybridisierung ebnet durch die existenziellen Gefühle der Unsicherheit den Weg

\footnotetext{
${ }^{7}$ Bhabha verwendet den Begriff ,große Erzählung“ in Anlehnung an Jean-François Lyotard (1924-1998).
} 
zur Reartikulierung der Machtverhältnisse im Ankunftskontext; jener „Wahrheitsregimes“, die durch „De-Platzierung“ der Wahrheit seitens des unterdrückten Menschen zu Ungewissheiten führen:

„Im Zwischenraum zwischen diesen beiden treibt die Zeit einer kolonialen Paradoxie in jenen widersprüchlichen Aussagen subordinierter Macht ihr Spiel. Denn die Wiederholung des ,Selben' kann in Wirklichkeit seine eigene De-plazierung sein, kann die kulturelle Autorität genau in dem Moment, in dem sie sich äußert, in ihren eigenen Un-sinn verwandeln. Denn im psychoanalytischen Sinne bedeutet ,Imitieren Anklammern an die Verleugnung der Grenzen des Ego; ,Identifizieren“ bedeutet Anpassung auf dem Weg über den Konflikt. Gerade aus dem Raum zwischen diesen Polen, wo der Buchstabe des Gesetzes nicht als Zeichen zugewiesen wird, kehrt das Doppel der Kultur auf unheimliche Weise - weder als das eine noch als das andere, das herrische Selbst und seine soziale Souveränität dem Verlust preiszugeben. Wenn dieser Moment intellektueller und psychischer ,Unsicherheit" erreicht ist, kann die Repräsentation die Autorität der Kultur nicht mehr garantieren, und die Kultur bietet keine Garantie mehr dafür, daß sie ihre ,menschlichen' Subjekte als Zeichen des Menschseins autorisiert“ (Bhabha 2000: 203).

Die Dimension des „Unheimlichen“ inmitten eines paradoxen Machtspiels zeigt sich deutlich in der Ambivalenz der Hybridität. Hier geschieht insofern etwas „Unheimliches“, als die Spaltung von Ghasems Selbst sich ebenso gegen das Selbst der dominanten Gesellschaft richtet. Auf diese Weise erlebt nicht nur Ghasem, sondern auch die deutsche Gesellschaft eine Selbstspaltung, die durch eine imaginäre Identifikation lanciert wird. Ghasem de-platziert mit seiner Narration unbewusst die Machtverhältnisse im Ankunftskontext. Verletzung, Verunsicherung, Kränkung und Ent-Selbstung bilden die Hauptdimensionen dieser entzweienden Verkennung. Die Autorität der dominanten Gesellschaft wird infolge der kulturellen Bedeutungsverschiebungen von einer radikalen Angst der Selbstauflösung geprägt sein. Das „Unheimliche“ ist anders ausgedrückt die „Doppel-Einschreibung“ (Bhabha 2000: 202) Ghasems mit unvereinbaren Prinzipien.

Zwei weitere Gesichtspunkte stehen nach dieser Deutung mit Ghasems Ambivalenz im Zusammenhang: die Unsichtbarkeit und die Sprachlosigkeit des gefangenen Menschen auf der sozialen Ebene. Die Unsichtbarkeit ergibt sich aus der Isolation der Angehörigen Ghasems. Er ist demnach völlig isoliert, obwohl er im Vergleich zu anderen Asylbewerber*innen sehr gut ,integriert“ zu sein scheint (zum Beispiel durch regelmäßigen Besuch von Sprach- und Ausbildungskursen). Die Sprachlosigkeit bezieht sich auf die allgemeine Position von Ghasem, der nicht über die Ressourcen verfügt, sich selbst zu artikulieren und zu erklären. Hier lässt sich das „stumme Bewußtsein“ Hegels (Abschn.4.7) im Individuum erkennen, das sich der vorgegebenen Rolle unterwirft. Ghasem ist nicht nur unfähig, sich zu 
artikulieren, er verliert zudem die Fähigkeit, durch andere anerkennend adressiert zu werden. Solche Prozesse der sprachlichen Unfähigkeit resultieren im Ausschluss sozialer Anerkennungskreisläufe (Bauman 2005). Die Energien, die ihm helfen könnten, sein wahres Selbst zu behaupten, werden Stück für Stück beschnitten, so dass er aus der Bedrängnis heraus ein Drama inszeniert, das ihm den soliden Halt für sein Selbst wiederherstellt. Ghasems Fall zeigt, wie lokale agency in Form strategischer Anpassungsmechanismen im liminalen Dritten Raum herangebildet wird. Die Ressentiments in Ghasem nach der schweren Kränkung durch die Angriffe der Institution in Form von entmündigenden Praktiken bilden den Antrieb seiner Narration. Kultur offenbart sich in diesem Zuge als eine lebendige, undurchschaubare und vor allem konstruierte Überlebensstrategie (Bhabha 2000: 257). 


\subsection{Intermezzo: „Die ganzen schwangeren Flüchtlingsfrauen!"}

Eine Vielzahl der in der Institution Asyl Bediensteten ist sich sicher, „die Flüchtlinge" dächten, ein in Deutschland geborenes Kind würde von den Behörden als unumstößliches Abschiebehindernis interpretiert. Über diese Annahme spotten sie offen. Ganze Kaffee- und Rauchpausen werden damit zugebracht, die „Tricks der Flüchtlinge“ zu diskutieren und zu beurteilen. Die ,vielen schwangeren Flüchtlingsfrauen“ bilden häufig ein Topthema auf den Raucherbalkonen des Landesamtes. Die lockere Atmosphäre dort eignet sich gut, um halb scherzend, halb im Ernst Dingen nachzugehen, die ich im Feld aufschnappe. Bei solchen Plaudereien erhalte ich in der Regel wertvolle Informationen. So lerne ich die Sachbearbeiterin Beate S. kennen. Sie ist überzeugt, dass die „ganzen Flüchtlinge“ sie hereinlegen würden. Sehr oft wiederholt sie den Satz „denken die, wir sind blöd?“", wenn es um „schwangere Flüchtlingsfrauen“ geht. Dabei wird sie aufbrausend. Sie berichtet, wie sie täglich mit ausgefuchsten Asylbewerber*innen konfrontiert sei, die nur betrügen wollten, natürlich des Geldes wegen. Ich nicke, „stelle mich dumm“ (siehe dazu die methodologischen Reflexionen) und versuche in dieser Situation so neutral wie möglich zu bleiben. Beistehende Bedienstete pflichten ihr bei, indem sie mit ernster Miene und hochgezogenen Augenbrauen nicken. Zwischendurch erklärt Beate S. ihre grundsätzliche Neutralität „den Anderen“ gegenüber, um sicherzugehen, dass ich mich nicht angegriffen fühle, indem sie plötzlich innehält und sagt: „Ich hab ja nix gegen Ausländer.“ Das wohl oder übel folgende "Aber" bettet sie in Narrative, die negative Eigenschaften der besagten Gruppe herausstellen. Später in ihrem Büro, das sie sich mit einer Kollegin teilt, inspiziere ich die Aktenberge, die in allen Ecken des Raums stehen und, wie ich erfahre, von niemandem gelesen werden. In diesen Akten finden sich etliche Bescheinigungen und Atteste von Asylbewerber*innen, die angesichts medizinischer Befunde eine bessere Unterkunft fordern - unter ihnen auch Untersuchungsergebnisse der „schwangeren Flüchtlingsfrauen“. Da alle im Amt wissen, dass Forderungen nach besseren Unterkünften utopisch sind, stapeln sich die Atteste ungelesen in den Räumlichkeiten der Behörde. Dafür sei einfach keine Zeit. Beate setzt sich in ihren Bürostuhl und dreht gleich am Radio herum, bis deutsche Popmusik ertönt. Es sei doch klar, dass „die alle eine Wohnung haben“" möchten, aber wer denke denn in dieser Situation 
an „die Deutschen“? Sie berichtet mir weiter von enormen Rechnungen von Krankenhäusern, auf die der Berliner Senat nun mal nicht eingestellt gewesen sei. Allein die Kosten für ,die vielen drogensüchtigen und schwangeren Flüchtlinge“" seien unfassbar hoch. Das Landesamt stottere derzeit noch seine Schulden bei diversen Berliner Hotels und Hostels ab. In letzter Zeit seien ungewöhnlich viele Fälle von Sozialbetrug aufgedeckt worden, weshalb Beate eine Menge zusätzlicher Arbeit am Hals habe. Sie verweist auf Pannen der Software, die im Landesamt verwendet wird. Die Software habe regelmäßig Ausfälle und scheine gänzlich ungeeignet zu sein, Asylbewerber*innen schnell und effektiv zu betreuen. Aufgrund dieser Ausfälle seien einige Zahlungen doppelt bzw. dreifach getätigt worden. Dessen ungeachtet seien immer mehr Fälle von Sachschäden in Unterkünften entstanden, für die Asylbewerber*innen verantwortlich gemacht würden. Man müsse deshalb „,mal streng sein mit denen“. Immer wieder rechtfertigt sich Beate S. vor mir. Die technischen Probleme hingen auch teils mit dem Umstand zusammen, dass im Landesamt alles mündlich kommuniziert werde. Konkrete, schriftliche Anweisungen existierten nicht. Im Laufe der Forschung stellt sich heraus, dass die Mehrheit der Sachbearbeiter*innen die fehlenden schriftlichen Anweisungen während aller Prozesse im Amt und die daraus resultierenden ständigen beliebigen Veränderungen in der Handhabe von Flüchtlingsangelegenheiten als Manifestationen des „Staatsversagens" ${ }^{\text {“8 }}$ betrachtet. Daraus spinnen sich Narrative zur Konsolidierung eigener Interessen und Meinungen, die zumeist darauf abzielen, die Geflüchteten zu inferiorisieren. Die beim Personal erkennbare Tendenz zur Erziehung der „Anderen“ in Denk- und Verhaltensweisen spiegelt diesen Umstand wider. Begleitet und unterstützt wird dies von rechtfertigenden Narrativen, die Gefühlen der Missgunst den Weg ebnen. Aufkommender Neid auf Asylbewerber*innen wird beispielsweise abgesehen vom Narrativ des Staatsversagens durch die Annahme bestärkt, Geflüchtete würden im Vergleich zu deutschen Bedürftigen zu Unrecht vom Staat bevorzugt, da sie ,alles“9 umsonst erhielten. In all diesen Erzählungen geht es primär um die Stärkung der eigenen Position,

${ }^{8}$ Dieser Begriff ist auf Seiten der Sachbearbeiter*innen recht beliebt.

${ }^{9}$ Gemeint sind vor allem Sozial- und Sachleistungen. 
die gegenüber den Asylbewerber*innen als überlegen dargestellt wird. Auf Seiten des Personals entwickelt sich so die Bereitschaft, die „Anderen“ im Geiste der Erziehung eines unterlegenen Individuums zu behandeln. Dies sorgt wiederum für Verbitterung und Verwirrung bei Geflüchteten, die eine andere Atmosphäre im Zielland ihrer Flucht erwartet haben. Mitten im Gespräch mit Beate streckt der Abteilungsleiter seinen Kopf ins Zimmer und ruft:

„Ab sofort keine Kohle mehr für Sommerbekleidung. Sagt denen einfach, die sollen den Antrag in drei Wochen nochmal stellen, dann is' es zu spät. Dann gibt et nix mehr!“

Kichernd entfernt er sich. Meine Anwesenheit bleibt - wenig überraschend unbemerkt. Kurz nach dem Auftritt des Abteilungsleiters verlasse ich das Büro.

Open Access Dieses Kapitel wird unter der Creative Commons Namensnennung 4.0 International Lizenz (http://creativecommons.org/licenses/by/4.0/deed.de) veröffentlicht, welche die Nutzung, Vervielfältigung, Bearbeitung, Verbreitung und Wiedergabe in jeglichem Medium und Format erlaubt, sofern Sie den/die ursprünglichen Autor(en) und die Quelle ordnungsgemäß nennen, einen Link zur Creative Commons Lizenz beifügen und angeben, ob Änderungen vorgenommen wurden.

Die in diesem Kapitel enthaltenen Bilder und sonstiges Drittmaterial unterliegen ebenfalls der genannten Creative Commons Lizenz, sofern sich aus der Abbildungslegende nichts anderes ergibt. Sofern das betreffende Material nicht unter der genannten Creative Commons Lizenz steht und die betreffende Handlung nicht nach gesetzlichen Vorschriften erlaubt ist, ist für die oben aufgeführten Weiterverwendungen des Materials die Einwilligung des jeweiligen Rechteinhabers einzuholen.

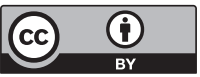

